\title{
Safety and efficacy profile of lenvatinib in cancer therapy: a systematic review and meta-analysis
}

\author{
Chenjing Zhu ${ }^{1, *}$, Xuelei Ma ${ }^{1, *}$, Yuanyuan $\mathrm{Hu}^{2}$, Linghong Guo ${ }^{2}$, Bo Chen ${ }^{3}$, Kai Shen ${ }^{1}$, \\ Yue Xiao ${ }^{2}$ \\ ${ }^{1}$ State Key Laboratory of Biotherapy and Cancer Center, West China Hospital, Sichuan University, and Collaborative Innovation \\ Center for Biotherapy, Chengdu 610041, PR China \\ ${ }^{2}$ West China School of Medicine, West China Hospital, Sichuan University, Chengdu 610041, PR China \\ ${ }^{3}$ Department of Oncology, The First People's Hospital of Chengdu City, Chengdu 610041, PR China \\ *These authors have contributed equally to this work
}

Correspondence to: Xuelei Ma, email: drmaxuelei@gmail.com

Keywords: safety, efficacy, lenvatinib, cancer, meta-analysis

Received: January 30, $2016 \quad$ Accepted: May 17, 2016

Published: June 14, 2016

\section{ABSTRACT}

To systematically review the safety and efficacy of lenvatinib in the treatment of patients, we retrieved all the relevant clinical trials on the adverse events (AEs) and survival outcomes of lenvatinib through PubMed, Medline, Embase, Web of Science and Cochrane Collaboration's Central register of controlled trial. Fourteen eligible studies involving a total of $\mathbf{9 7 8}$ patients were included in our analysis. The most common all-grade AEs observed in patients treated with lenvatinib were hematuria $(56.6 \%)$, fatigue $(52.2 \%)$ and decreased appetite $(50.5 \%)$. The most frequently observed grade $\geq 3$ AEs were thrombocytopenia (25.4\%), hypertension $(17.7 \%)$ and edema peripheral $(15.5 \%)$. The incidences of both all-grade and high-grade hypertension were significantly increased. Meanwhile, the controlled trial suggested that progression free survival (PFS) was significantly longer in the lenvatinib group than the placebo group. Subgroup analyses showed that mean PFS for renal cell carcinoma was $10.933 \pm 1.828$ months $(95 \%$ CI $7.350-14.515, p<0.001)$, and that for thyroid cancer was $18.344 \pm 0.083$ months (95\% CI 18.181-18.506, $p<0.001$ ). In conclusion, lenvatinib is an effective agent in thyroid cancer. Early monitoring and effective management of side effects are crucial for the safe use of this drug.

\section{INTRODUCTION}

Angiogenesis is critical for the local invasion and progression of tumor cells [1]. The aberrant formation and proliferation of blood vessels is due to an imbalance in pro- and anti-angiogenic factors, with the first weighing more [2]. Vascular endothelial growth factor (VEGF), fibroblast growth factor (FGF), platelet-derived growth factor (PDGF) and epidermal growth factor (EGF) are several positive regulators of angiogenesis [3]. Over the last decade, multi-targeted tyrosine kinase inhibitors (TKIs) have been developed and approved in clinical oncology practice [4].

Lenvatinib (E7080) is an oral, multi-targeted tyrosine kinase inhibitor of VEGFR, FGFR, PDGFR and
RET $[5,6]$. With its anti-angiogenic activity, and a direct effect on tumor cells by preventing relevant signaling pathways [6-8], lenvatinib has been observed to have promising effects in clinical trials for thyroid cancer [9, 10]. In February 2015, US FDA has approved lenvatinib for the treatment of locally recurrent or metastatic, progressive, radioactive iodine-refractory differentiated thyroid cancer (RR-DTC) [9].

Lenvatinib has brought clinical benefits for patients, but adverse events (AEs) are inevitable such as hypertension, fatigue, proteinuria, nausea, decreased weight and abdominal pain, which may decrease the quality of life of patients and influence their acceptance of treatment [11, 12]. Therefore, we conducted a meta-analysis to estimate various AEs and clinical benefits of lenvatinib. 


\section{RESULTS}

\section{Literature search results}

We ran an initial broad search that yielded 422 unique articles after deletion of duplicates. After title and abstract screening, 344 were excluded since they were narrative review articles or interviews, or completely not associated with clinical assessment of lenvatinib. Forty were further excluded for they were conference abstracts based on published clinical trials, leaving 38 potentially relevant studies for full review. After estimating the full texts of these articles, 24 articles were ruled out for insufficient information. Ultimately, 14 eligible studies [13-26] involving a total of 978 patients met our metaanalysis criteria. Two articles $[22,26]$ with the same first author which had different study designs were both included in our study, one was a phase II trial, and the other was a phase III, randomized multicenter study. No additional unpublished trials were added to the literature search results. A flow diagram of the trial selection process is provided in Figure 1.

\section{Study characteristics}

Of the studies that were included in the final analysis, 3 studies were based on thyroid cancer patients, 5 evaluated advanced solid tumors, 1 evaluated non-small-cell lung cancer, 1 was based on melanoma, 2 were performed on metastatic renal cell carcinoma, 1 was on advanced hepatocellular carcinoma and 1 on healthy adults. Schlumberger M [22] compared lenvatinib with placebo in radioiodine-refractory thyroid cancer patients, and Motzer RJ [19] used lenvatinibeither in combination with everolimus or as a single agent in patients with metastatic renal cell carcinoma. The characteristics of each trial are summarized in Table 1.

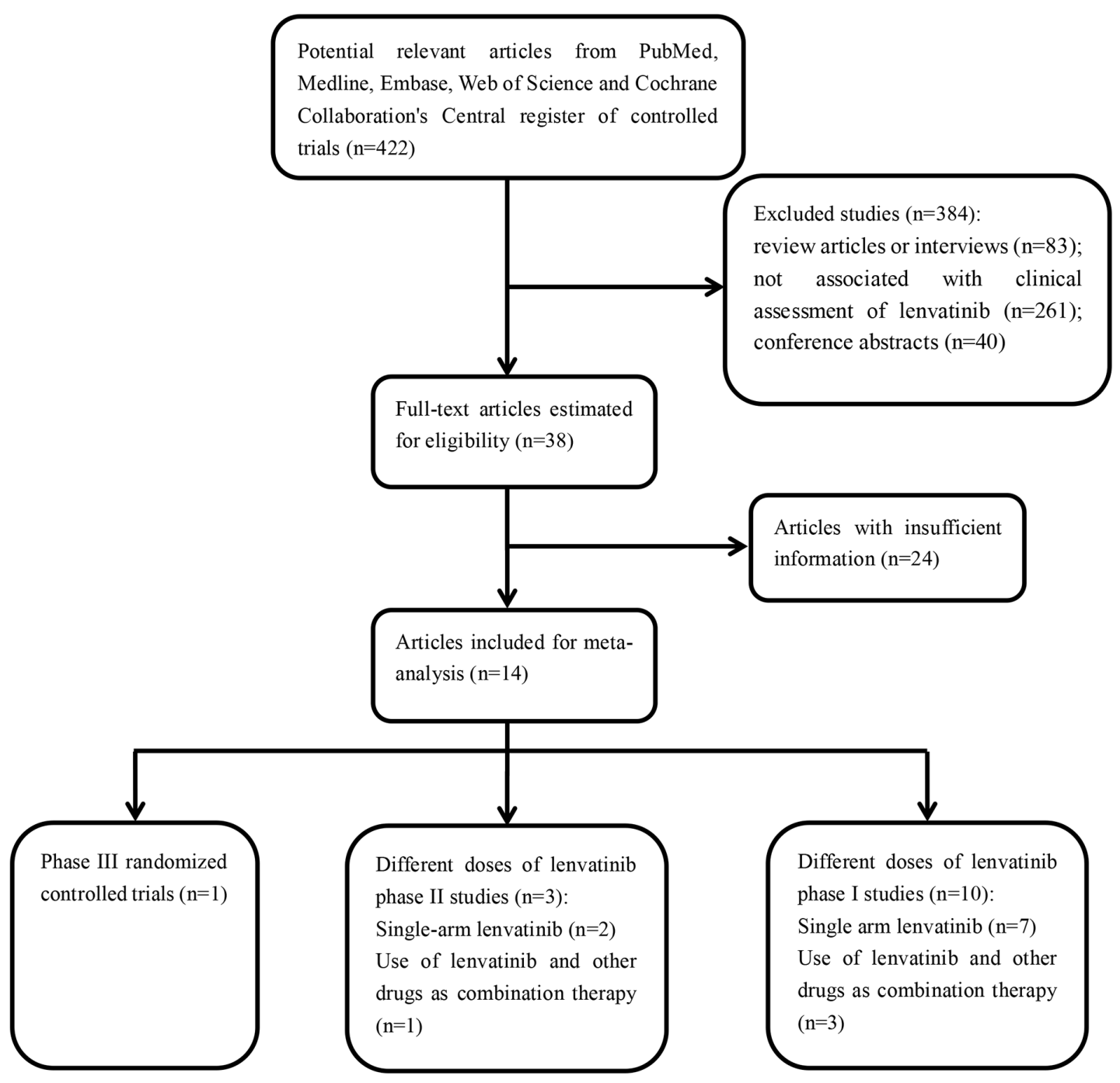

Figure 1: Flow diagram of the literature search and selection process. 
Table 1: Basic characteristics of the included articles

\begin{tabular}{|c|c|c|c|c|c|c|c|c|c|c|}
\hline \multirow[t]{2}{*}{ First author } & \multirow[t]{2}{*}{ Year } & \multirow[t]{2}{*}{ Phase } & \multirow[t]{2}{*}{ Sample size } & \multicolumn{2}{|c|}{ Gender } & \multirow[t]{2}{*}{ Age } & \multirow[t]{2}{*}{ Region } & \multirow[t]{2}{*}{ Histology } & \multirow{2}{*}{$\begin{array}{c}\text { Treatment } \\
\text { arm }\end{array}$} & \multirow{2}{*}{$\begin{array}{c}\text { Treatment } \\
\text { regimen }\end{array}$} \\
\hline & & & & Male & Female & & & & & \\
\hline $\begin{array}{l}\text { Schlumberger } \\
\mathrm{M}^{1}\end{array}$ & 2015 & II & 59 & 37 & 22 & Mean 52 & $\begin{array}{c}\text { United } \\
\text { States, } \\
\text { United } \\
\text { Kingdom, } \\
\text { Australia, } \\
\text { France, Italy, } \\
\text { and Poland }\end{array}$ & MTC or DTC & Lenvatinib & $\begin{array}{l}\text { Lenvatinib } 24 \\
\text { mg Qd, 28-day } \\
\text { cycles }\end{array}$ \\
\hline Hong DS ${ }^{1}$ & 2015 & I & 77 & 40 & 37 & $\begin{array}{l}\text { Median(range) } \\
61.0(28-85)\end{array}$ & USA & $\begin{array}{l}\text { Advanced } \\
\text { solid tumor; } \\
\text { Melanoma }\end{array}$ & Lenvatinib & $\begin{array}{c}\text { Lenvatinib } \\
0.1-3.2 \mathrm{mg} \text { Bid } \\
(\mathrm{n}=18) ; 3.2-12 \\
\mathrm{mg} \mathrm{Bid}(\mathrm{n}=33) ; \\
10 \mathrm{mg} \mathrm{Bid} \\
(\mathrm{n}=26)\end{array}$ \\
\hline Cabanillas ME & 2015 & II & 58 & 34 & 24 & $\begin{array}{l}\text { Median(range) } \\
63(34-77)\end{array}$ & USA & RR-DTC & Lenvatinib & $\begin{array}{l}\text { Lenvatinib } 24 \\
\text { mg Qd, 28-day } \\
\text { cycles }\end{array}$ \\
\hline $\begin{array}{l}\text { Schlumberger } \\
\mathrm{M}^{2}\end{array}$ & 2015 & III & $\begin{array}{l}\text { 392(lenvatinib: } \\
\text { n=261, } \\
\text { placebo: } \\
\text { n=131) }\end{array}$ & 125,75 & 136,56 & $\begin{array}{l}\text { Lenvatinib: } \\
\text { median } 64, \\
\text { placebo: } \\
\text { median } 61\end{array}$ & USA & RR-DTC & $\begin{array}{l}\text { Lenvatinib/ } \\
\text { placebo }\end{array}$ & $\begin{array}{l}\text { Lenvatinib } 24 \\
\text { mg Qd, 28-day } \\
\text { cycles/placebo }\end{array}$ \\
\hline Dubbelman AC & 2014 & I & 6 & 3 & 3 & $\begin{array}{c}\text { Median(range) } \\
49(34-64)\end{array}$ & Netherlands & $\begin{array}{l}\text { Advanced } \\
\text { solid tumors; } \\
\text { lymphomas }\end{array}$ & Lenvatinib & $\begin{array}{c}\text { Lenvatinib } 24 \\
\text { mg Qd, 28-day } \\
\text { cycles }\end{array}$ \\
\hline Shumaker RC & 2014 & I & 15 & 11 & 4 & $\begin{array}{l}\text { Median(range) } \\
31(20-49)\end{array}$ & USA & Healthy adult & $\begin{array}{l}\text { Lenvatinib } \\
\text { plus } \\
\text { rifampicin }\end{array}$ & $\begin{array}{c}\text { Lenvatinib } \\
24 \mathrm{mg} / \\
\text { coadministrate } \\
\text { rifampicin } 600 \\
\text { mg }\end{array}$ \\
\hline Molina AM & 2013 & $\mathrm{Ib}$ & 20 & 14 & 6 & $\begin{array}{l}\text { Mean(SD) } \\
58.4(6.29)\end{array}$ & Finland & $\begin{array}{l}\text { Metastatic } \\
\text { renal cell } \\
\text { carcinoma }\end{array}$ & $\begin{array}{l}\text { Lenvatinib } \\
\text { plus } \\
\text { everolimus }\end{array}$ & $\begin{array}{l}\text { Lenvatinib } \\
{[12 \mathrm{mg}(\mathrm{n}=} \\
7) ; 18 \mathrm{mg}(\mathrm{n} \\
=11) ; 24 \mathrm{mg} \\
(\mathrm{n}=2)] \text { plus } \\
\text { everolimus } 5 \\
\text { mg, 28-day } \\
\text { cycles }\end{array}$ \\
\hline Boss DS & 2012 & I & 82 & 43 & 39 & $\begin{array}{l}\text { Median(range) } \\
54(25-84)\end{array}$ & USA & $\begin{array}{c}\text { Advanced } \\
\text { solid tumours }\end{array}$ & Lenvatinib & $\begin{array}{l}\text { Dose cohorts } \\
\text { from } 0.2 \text { to } 32 \\
\text { mg, } 28 \text {-day } \\
\text { cycles }\end{array}$ \\
\hline Nishio M & 2013 & I & 28 & 21 & 7 & $\begin{array}{c}\text { Mean(range) } \\
56.4(38-73)\end{array}$ & Japan & $\begin{array}{l}\text { Non-small- } \\
\text { cell lung } \\
\text { cancer }\end{array}$ & Lenvatinib & $\begin{array}{c}\text { Lenvatinib 4/6 } \\
\text { mg Bid }\end{array}$ \\
\hline Yamada K & 2011 & I & 27 & 10 & 17 & $\begin{array}{l}\text { Median(range) } \\
53(26-70)\end{array}$ & Japan & $\begin{array}{c}\text { Advanced } \\
\text { solid tumours }\end{array}$ & Lenvatinib & $\begin{array}{l}\text { From } 0.5 \text { to } 1 \text {, } \\
2,4,6,9,13, \\
16 \text {, and } 20 \mathrm{mg} \\
\text { Bid }\end{array}$ \\
\hline Nakamichi S & 2015 & I & 9 & 2 & 7 & $\begin{array}{c}\text { Median(range) } \\
41(30-59)\end{array}$ & Japan & $\begin{array}{c}\text { Advanced } \\
\text { solid tumours }\end{array}$ & Lenvatinib & $\begin{array}{c}\text { Lenvatinib [20 } \\
\mathrm{mg}(\mathrm{n}=3) ; 24 \\
\mathrm{mg}(\mathrm{n}=6)] \\
\text { 28-day cycles }\end{array}$ \\
\hline
\end{tabular}




\begin{tabular}{|c|c|c|c|c|c|c|c|c|c|c|}
\hline \multirow[t]{2}{*}{ First author } & \multirow[t]{2}{*}{ Year } & \multirow[t]{2}{*}{ Phase } & \multirow[t]{2}{*}{ Sample size } & \multicolumn{2}{|c|}{ Gender } & \multirow[t]{2}{*}{ Age } & \multirow[t]{2}{*}{ Region } & \multirow[t]{2}{*}{ Histology } & \multirow{2}{*}{$\begin{array}{l}\text { Treatment } \\
\text { arm }\end{array}$} & \multirow{2}{*}{$\begin{array}{l}\text { Treatment } \\
\text { regimen }\end{array}$} \\
\hline & & & & Male & Female & & & & & \\
\hline Hong DS ${ }^{2}$ & 2015 & $\mathrm{Ib}$ & 32 & 20 & 12 & $\begin{array}{c}\text { Median(range) } \\
57.5(24-81)\end{array}$ & USA & $\begin{array}{l}\text { Advanced } \\
\text { melanoma }\end{array}$ & $\begin{array}{l}\text { Lenvatinib } \\
\text { plus TMZ }\end{array}$ & $\begin{array}{l}\text { Dose Level } \\
\text { (DL)1: } \\
\text { lenvatinib } 20 \\
\text { mg, TMZ } 100 \\
\text { mg/m2; DL2: } \\
\text { lenvatinib } 24 \\
\text { mg, TMZ } 100 \\
\text { mg/m2; DL3: } \\
\text { lenvatinib } 24 \\
\text { mg, TMZ 150 } \\
\text { mg/m2, 28-day } \\
\text { cycles }\end{array}$ \\
\hline Ikeda M & 2015 & I & 20 & 17 & 3 & $\begin{array}{l}\text { Median(range) } \\
63.5(47-74)\end{array}$ & Japan & $\begin{array}{l}\text { Advanced } \\
\text { hepatocellular } \\
\text { carcinoma }\end{array}$ & Lenvatinib & $\begin{array}{l}\text { Lenvatinib } 8 \\
\mathrm{mg}, 12 \mathrm{mg}, 16 \\
\mathrm{mg}, 25 \mathrm{mg} \mathrm{Qd} \text {, } \\
\text { 4-week cycles }\end{array}$ \\
\hline Motzer RJ & 2015 & II & $\begin{array}{l}\text { 153(lenvatinib: } \\
\text { n=52, } \\
\text { everolimus: } \\
n=50 \text {, } \\
\text { lenvatinib plus } \\
\text { everolimus: } \\
n=51 \text { ) }\end{array}$ & 112 & 41 & $\begin{array}{l}\text { Median(range) } \\
59(37-77)\end{array}$ & $\begin{array}{c}\text { Czech } \\
\text { Republic, } \\
\text { Poland, } \\
\text { Spain, the } \\
\text { UK, and the } \\
\text { USA }\end{array}$ & $\begin{array}{l}\text { Metastatic } \\
\text { renal cell } \\
\text { carcinoma }\end{array}$ & Lenvatinib & $\begin{array}{l}\text { Lenvatinib } \\
\text { (24 mg/day), } \\
\text { everolimus ( } 10 \\
\text { mg/day), or } \\
\text { lenvatinib plus } \\
\text { everolimus } \\
\text { ( } 18 \mathrm{mg} / \text { day } \\
\text { and } 5 \mathrm{mg} / \text { day, } \\
\text { respectively), } \\
\text { 28-day cycles }\end{array}$ \\
\hline
\end{tabular}

MTC: medullary thyroid cancer; RR-DTC: radioiodine-refractory, differentiated thyroid cancer Schlumberger $\mathrm{M}^{1}$ and ${ }^{2}$ : the former was a single-arm trial, while the latter was a controlled trial

\section{Adverse drug reactions analyses}

To evaluate the safety of lenvatinib, we calculated the rates of all-grade and grade 3 or more serious adverse events in the overall population. In single-arm trials with all-grade AEs, homogeneity existed in upper abdominal pain, arthralgia, constipation and peripheral edema etc., which were further analysed with a fixed-effects model (Figure 2a, Table 2). Others were analysed using a random-effects model (Figure 2b, Table 2). Hematuria (56.6\%, 95\% CI $0.193-0.877)$, fatigue $(52.2 \%, 95 \%$ CI 0.384-0.657), palmar-plantar erythrodysesthesia syndrome $(47.2 \%, 95 \%$ CI $0.201-0.761)$, hypertension $(47.0 \%, 95 \%$ CI $0.354-0.589)$ and diarrhea $(46.2 \%, 95 \%$ CI 0.362-0.605) were common in a random-effects model (Figure 2b, Table 2). Increased alanine aminotransferase occurred in $42 \%$ of the patients using a fixed-effects model $(42.0 \%, 95 \%$ CI $0.294-0.556)$. The most frequent grade $\geq$ 3 treatment-related adverse events were thrombocytopenia (25.4\%, 95\% CI 0.055-0.665, random model), hypertension $(17.7 \%, 95 \%$ CI $0.102-0.289$, random model), peripheral edema ( $15.5 \%, 95 \%$ CI $0.020-0.622$, random model) and increased aspartate aminotransferase (12.6\%, 95\% CI 0.061-0.242, fixed model) (Figure 2c, 2d, Table 2).

\section{Survival outcomes and subgroup analysis}

The efficacy analysis of lenvatinib was mainly based on the controlled trial of lenvatinib in patients with thyroid cancer [22]. The median progression-free survival was 18.3 months in the lenvatinib group and 3.6 months in the placebo group (hazard ratio for progression or death 0.21 , $99 \%$ CI 0.14-0.31, P < 0.001). In addition, Motzer RJ [19] reported that median PFS was 7.4 months $(95 \%$ CI 5.610.2) for single-agent lenvatinib in patients with metastatic renal cell carcinoma and 5.5 months (95\% CI 3.5-7.1) for single-agent everolimus, representing the significantly prolonged PFS of lenvatinib compared with everolimus alone (HR 0.61, 95\% CI 0.38-0.98, $\mathrm{p}=0.048$ ). Seven trials $[13,15,16,19,22,25,26]$ reported encouraging response rates, median time to response, or PFS observed in patients with different types of tumors, demonstrating the antitumour efficacy of lenvatinib (Table 3). We further carried out subgroup analyses according to tumor types. Mean PFS for renal cell carcinoma was $10.933 \pm 1.828$ months (95\% CI 7.350-14.515, p < 0.001), and that for thyroid cancer was $18.344 \pm 0.083$ months (95\% CI 18.181-18.506, $\mathrm{p}<0.001$ ) (Table 4). Further large-scale studies are still needed to assess the PFS of patients with melanoma and non-small-cell lung cancer. 

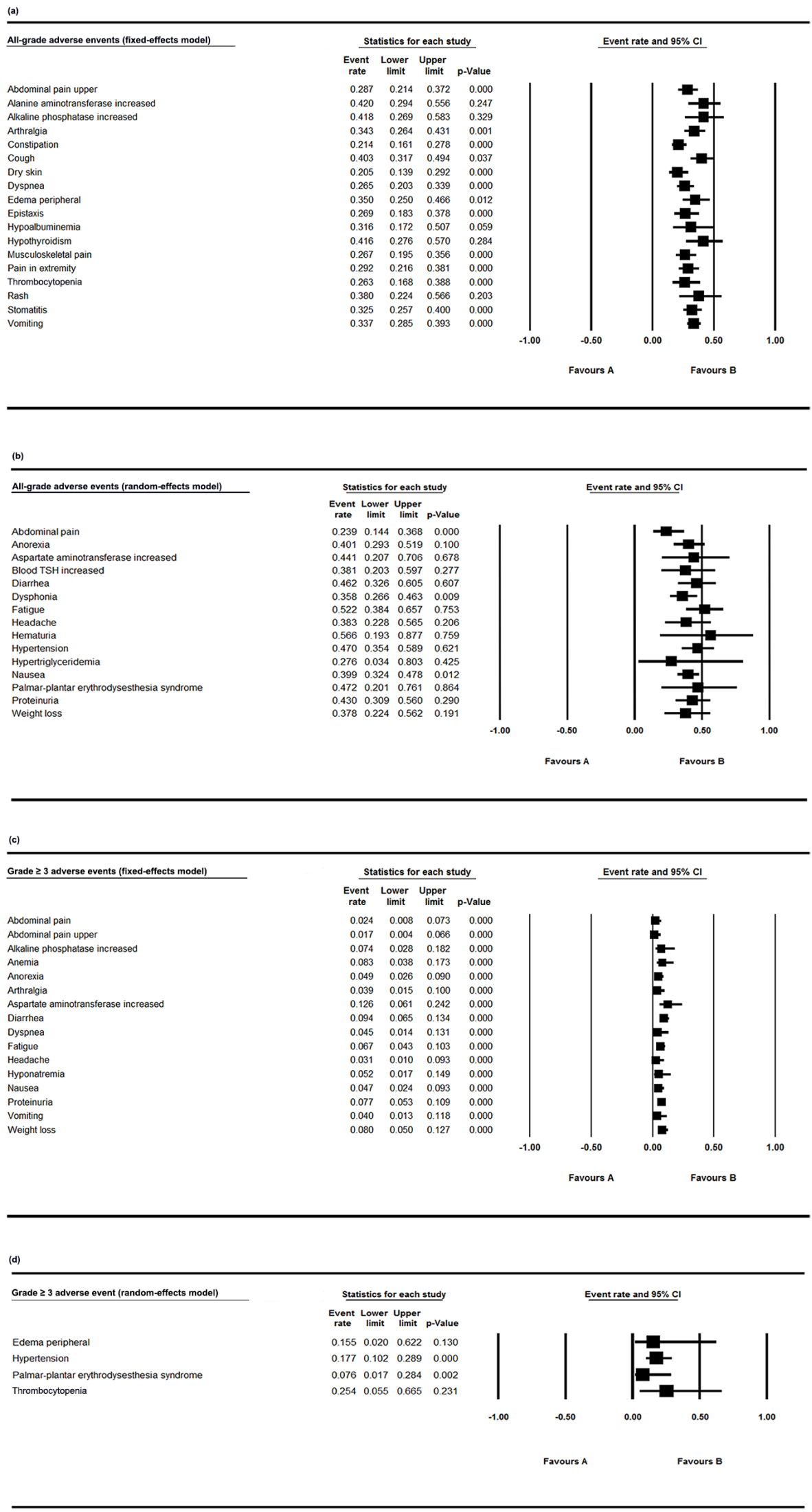

Figure 2: Forest plot of all-grade and grade $\geq \mathbf{3}$ AEs in single-arm trials. a. The all-grade adverse event rates and $95 \%$ CIs using a fixed-effects model; $\mathbf{b}$. The all-grade adverse event rates and $95 \%$ CIs using a random-effects model; $\mathbf{c}$. The grade $\geq 3$ adverse event rates and $95 \%$ CIs using a fixed-effects model; $\mathbf{d}$. The grade $\geq 3$ adverse event rates and $95 \%$ CIs using a random-effects model. 
Table 2: Summary results of the all-grade and grade $\geq 3$ adverse events (AEs) with $95 \%$ confidence intervals

\begin{tabular}{|c|c|c|}
\hline All-grade adverse events & Model & Event rate with $95 \% \mathrm{CI}$ \\
\hline Abdominal pain upper & Fixed model & $0.287(0.214-0.372)$ \\
\hline Alanine aminotransferase increased & Fixed model & $0.420(0.294-0.556)$ \\
\hline Alkaline phosphatase increased & Fixed model & $0.418(0.269-0.583)$ \\
\hline Arthralgia & Fixed model & $0.343(0.264-0.431)$ \\
\hline Constipation & Fixed model & $0.214(0.161-0.278)$ \\
\hline Cough & Fixed model & $0.403(0.317-0.494)$ \\
\hline Dry skin & Fixed model & $0.205(0.139-0.292)$ \\
\hline Dyspnea & Fixed model & $0.265(0.203-0.339)$ \\
\hline Edema peripheral & Fixed model & $0.350(0.250-0.466)$ \\
\hline Epistaxis & Fixed model & $0.269(0.183-0.378)$ \\
\hline Hypoalbuminemia & Fixed model & $0.316(0.172-0.507)$ \\
\hline Hypothyroidism & Fixed model & $0.416(0.276-0.570)$ \\
\hline Musculoskeletal pain & Fixed model & $0.267(0.195-0.356)$ \\
\hline Pain in extremity & Fixed model & $0.292(0.216-0.381)$ \\
\hline Thrombocytopenia & Fixed model & $0.263(0.168-0.388)$ \\
\hline Rash & Fixed model & $0.380(0.224-0.566)$ \\
\hline Stomatitis & Fixed model & $0.325(0.257-0.400)$ \\
\hline Vomiting & Fixed model & $0.337(0.285-0.393)$ \\
\hline Abdominal pain & Random model & $0.239(0.144-0.368)$ \\
\hline Anorexia & Random model & $0.401(0.293-0.519)$ \\
\hline Aspartate aminotransferase increased & Random model & $0.441(0.207-0.706)$ \\
\hline Blood TSH increased & Random model & $0.381(0.203-0.597)$ \\
\hline Diarrhea & Random model & $0.462(0.326-0.605)$ \\
\hline Dysphonia & Random model & $0.358(0.266-0.463)$ \\
\hline Fatigue & Random model & $0.522(0.384-0.657)$ \\
\hline Headache & Random model & $0.383(0.228-0.565)$ \\
\hline Hematuria & Random model & $0.566(0.193-0.877)$ \\
\hline Hypertension & Random model & $0.470(0.354-0.589)$ \\
\hline Hypertriglyceridemia & Random model & $0.276(0.034-0.803)$ \\
\hline Nausea & Random model & $0.399(0.324-0.478)$ \\
\hline Palmar-plantar erythrodysesthesia syndrome & Random model & $0.472(0.201-0.761)$ \\
\hline Proteinuria & Random model & $0.430(0.309-0.560)$ \\
\hline Weight loss & Random model & $0.378(0.224-0.562)$ \\
\hline Grade $\geq 3$ adverse events & Model & Event rate with $95 \%$ CI \\
\hline Abdominal pain & Fixed model & $0.024(0.008-0.073)$ \\
\hline Abdominal pain upper & Fixed model & $0.017(0.004-0.066)$ \\
\hline Alkaline phosphatase increased & Fixed model & $0.074(0.028-0.182)$ \\
\hline
\end{tabular}




\begin{tabular}{lcc}
\hline Grade $\geq \mathbf{3}$ adverse events & Model & Event rate with 95\% CI \\
\hline Anemia & Fixed model & $0.083(0.038-0.173)$ \\
Anorexia & Fixed model & $0.049(0.026-0.090)$ \\
Arthralgia & Fixed model & $0.039(0.015-0.100)$ \\
Aspartate aminotransferase increased & Fixed model & $0.126(0.061-0.242)$ \\
diarrhea & Fixed model & $0.094(0.065-0.134)$ \\
Dyspnea & Fixed model & $0.045(0.014-0.131)$ \\
Fatigue & Fixed model & $0.067(0.043-0.103)$ \\
Headache & Fixed model & $0.031(0.010-0.093)$ \\
Hyponatremia & Fixed model & $0.052(0.017-0.149)$ \\
Nausea & Fixed model & $0.047(0.024-0.093)$ \\
Proteinuria & Fixed model & $0.077(0.053-0.109)$ \\
Vomiting & Fixed model & $0.040(0.013-0.118)$ \\
Weight loss & Fixed model & $0.080(0.050-0.127)$ \\
Edema peripheral & Random model & $0.155(0.020-0.622)$ \\
Hypertension & Random model & $0.177(0.102-0.289)$ \\
Palmar-plantar erythrodysesthesia syndrome & Random model & $0.076(0.017-0.284)$ \\
Thrombocytopenia & Random model & $0.254(0.055-0.665)$ \\
\hline
\end{tabular}

Table 3: The median PFS of the included trials

\begin{tabular}{|c|c|c|c|c|c|c|}
\hline Study & $\begin{array}{l}\text { Sample } \\
\text { size }\end{array}$ & Tumor types & $\begin{array}{c}\text { Median PFS } \\
\text { (95\%CI) } \\
\text { (Months) }\end{array}$ & Mean & SD & $\begin{array}{c}\text { Overall } \\
\text { median OS }\end{array}$ \\
\hline \multirow[t]{2}{*}{ Boss DS 2012} & 9 & $\begin{array}{l}\text { renal cell } \\
\text { carcinoma }\end{array}$ & $15.9(9.3-18.63)$ & 14.93 & 2.75 & \\
\hline & 14 & melanoma & $\begin{array}{c}7.23(3.63- \\
12.63)\end{array}$ & 7.68 & 2.61 & \\
\hline $\begin{array}{l}\text { Schlumberger } \\
\mathrm{M}^{1} 2015\end{array}$ & 59 & MTC & $9.0(7-16.6)$ & 6.25 & 2.4 & 16.6 (16.4-NE) \\
\hline $\begin{array}{l}\text { Cabanillas ME } \\
2015\end{array}$ & 58 & RR-DTC & $12.6(9.9-16.1)$ & 12.8 & 1.55 & \\
\hline $\begin{array}{l}\text { Molina AM } \\
2014\end{array}$ & 20 & $\begin{array}{l}\text { renal cell } \\
\text { carcinoma }\end{array}$ & $11(5.23-14.87)$ & 10.525 & 2.41 & \\
\hline Nishio M 2013 & 28 & $\begin{array}{l}\text { non-small-cell } \\
\text { lung cancer }\end{array}$ & $9.0(6.5-9.5)$ & 8.5 & 0.75 & \\
\hline $\begin{array}{l}\text { Schlumberger } \\
\mathrm{M}^{2} 2015\end{array}$ & 392 & RR-DTC & $18.3(15.2-26)$ & 19.45 & 1.8 & \\
\hline Motzer RJ 2015 & 153 & $\begin{array}{l}\text { renal cell } \\
\text { carcinoma }\end{array}$ & $7.4(5.6-10.2)$ & 7.65 & 0.77 & \\
\hline
\end{tabular}

SD: Standard deviation estimation

MTC: medullary thyroid cancer

RR-DTC: radioiodine-refractory, differentiated thyroid cancer

Martin schlumberger ${ }^{1}$ and ${ }^{2}$ : the former was a single-arm trial, while the latter was a controlled trial 
Table 4: Subgroup analysis for survival outcomes

\begin{tabular}{|c|c|c|c|c|c|c|c|c|c|}
\hline \multirow[t]{2}{*}{ First author } & \multirow[t]{2}{*}{ Model } & \multirow[t]{2}{*}{ Mean } & \multirow{2}{*}{$\begin{array}{c}\text { Standard } \\
\text { error }\end{array}$} & \multirow[t]{2}{*}{ Variance } & \multicolumn{2}{|c|}{$95 \%$ CI } & \multirow[t]{2}{*}{ Z-Value } & \multirow[t]{2}{*}{ P-Value } & \multirow[t]{2}{*}{ Histology } \\
\hline & & & & & $\begin{array}{c}\text { lower } \\
\text { limit }\end{array}$ & $\begin{array}{l}\text { upper } \\
\text { limit }\end{array}$ & & & \\
\hline $\begin{array}{l}\text { Cabanillas ME } \\
2015\end{array}$ & & 12.800 & 0.204 & 0.041 & 12.401 & 13.199 & 62.892 & & RR-DTC \\
\hline $\begin{array}{l}\text { Schlumberger } \\
\mathrm{M}^{2} 2015\end{array}$ & & 19.450 & 0.091 & 0.008 & 19.272 & 19.628 & 213.933 & & RR-DTC \\
\hline Overall & Random & 18.344 & 0.083 & 0.007 & 18.181 & 18.506 & 220.987 & $<0.001$ & \\
\hline $\begin{array}{l}\text { Molina AM } \\
2014\end{array}$ & & 10.525 & 0.539 & 0.290 & 9.469 & 11.581 & 19.531 & & $\mathrm{RCC}$ \\
\hline Boss DS 2012 & & 14.930 & 0.917 & 0.840 & 13.133 & 16.727 & 16.287 & & $\mathrm{RCC}$ \\
\hline $\begin{array}{l}\text { Motzer RJ } \\
2015\end{array}$ & & 7.650 & 0.062 & 0.004 & 7.528 & 7.772 & 122.890 & & $\mathrm{RCC}$ \\
\hline Overall & Random & 10.933 & 1.828 & 3.341 & 7.350 & 14.515 & 5.981 & $<0.001$ & \\
\hline
\end{tabular}

MTC: medullary thyroid cancer

RR-DTC: radioiodine-refractory, differentiated thyroid cancer

RCC: Renal cell carcinoma

Schlumberger $\mathrm{M}^{1}$ and ${ }^{2}$ : the former was a single-arm trial, while the latter was a controlled trial

\section{Risk of bias and quality assessment}

The risk of bias and quality assessments of the included studies are outlined in Figure 3a, 3b. Overall, the quality of the studies was satisfactory.

\section{DISCUSSION}

To the best of our knowledge, this is the first study to evaluate both the safety and efficacy of the novel antitumor agent lenvantinib in different types of tumors systematically. The adverse events of lenvatinib were tyrosine kinase inhibitor-related and were also seen in other TKIs. In one meta-analysis [27], the VEGFRTKIs group (cediranib and axitinib) was associated with higher rates of diarrhea, fatigue, hypertension and thrombocytopenia compared with bevacizumab. Vandetanib [28], a dual VEGFR and EGFR inhibitor, yielded an improvement in PFS but more frequent grade 3 or greater hypertension. Although the incidence of hematuria was high, most people experienced low grade (grade 0) hematuria.

It should be noted that lenvatinib was associated with a significantly increased risk in all-grade $(47.0 \%)$ and high-grade (17.7\%) hypertension. The mechanism of lenvatinib-associated hypertension has not been clarified, and may be due to a possible perturbation of endothelial cell function in patients treated with VEGF-targeting agents [29]. It has been documented upon administration of bevacuzimab and cediranib, and several other inhibitors of the VEGF signalling pathway [30-32]. All of these suggest that patients who were administered lenvatinib should be monitored for high blood pressure, and managed with antihypertensive drugs or dose reductions when necessary.

Grade $\geq 3$ thrombocytopenia was experienced in about a quarter of patients. Through binding to PDGFR, PDGF promotes the recovery of platelets and the formation of bone marrow colony-forming unit-megakaryocyte [33, 34], thus the inhibition of PDGFR by lenvatinib might cause thrombocytopenia. Hematopoietic growth factors and transfusions [35] could be used to deal with persistent toxicities on platelets, but the effects of them on tumor cells remain to be explored.

In February 2015, US FDA has approved lenvatinib for the treatment of radioiodine-refractory thyroid cancer [9] based on the randomized controlled trial [22] included in our analysis. We find a similar mean PFS (18.344 \pm 0.083 months, 95\% CI 18.181-18.506, $\mathrm{p}<0.001$ ) for thyroid cancer in our pooled analysis. However, our results of adverse events (Figure 4a, 4b) are different, since the relatively larger sample size may allow us to better determine the AE values. Survival outcomes of other tumors are mainly based on phase I and phase II trials, and more subsequent randomized, controlled phase III trials are needed.

The dose of lenvatinib administered in patients with solid tumors varied in different situations, but in $8[15$, $16,18-20,22,23,26]$ of the 14 included studies, patients received lenvatinib at a daily dose of $24 \mathrm{mg}$ per day in 28 -day cycles, and two studies $[18,20]$ demonstrated that the 24-mg QD dose of lenvatinib was determined to be 

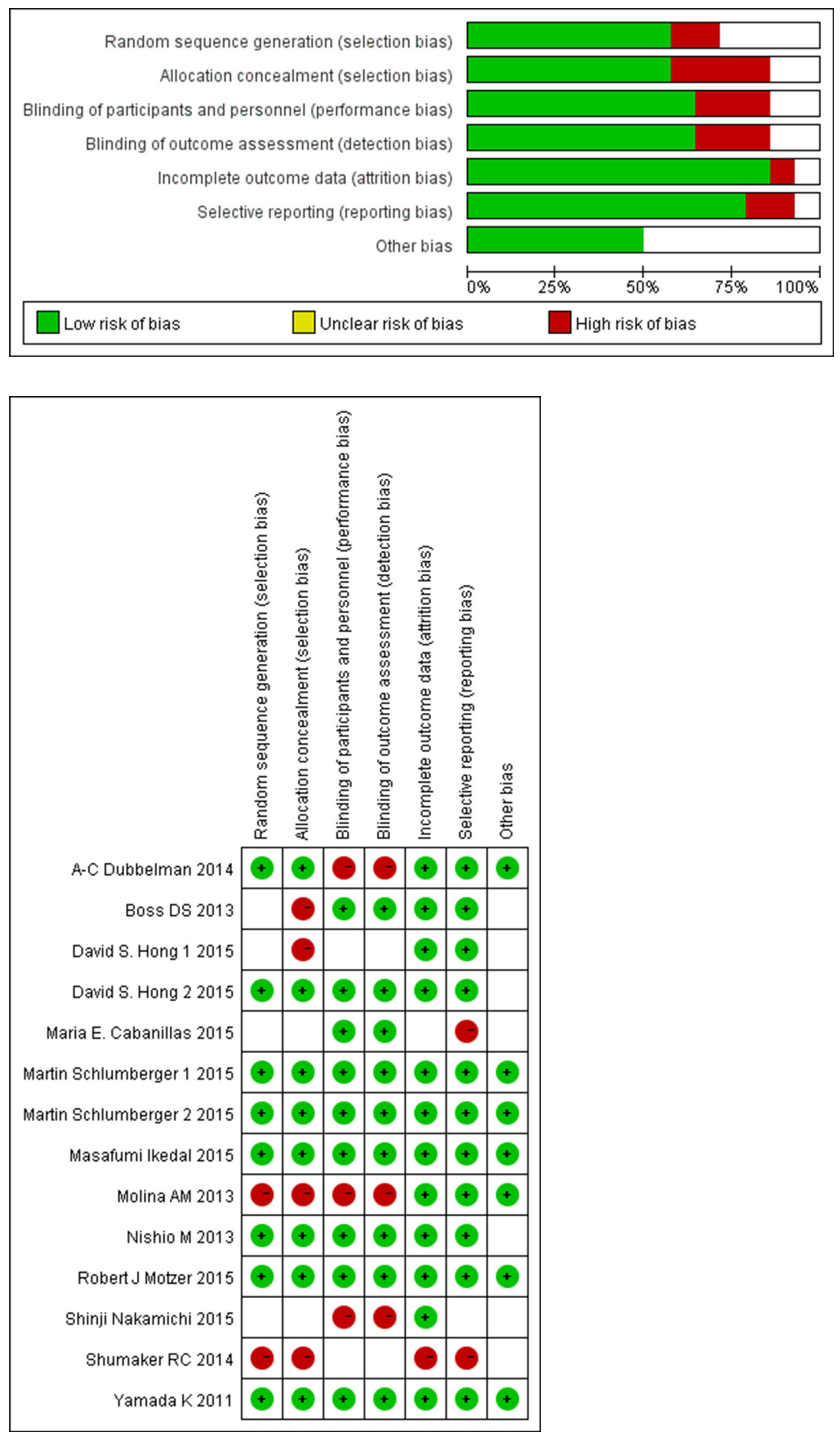

Figure 3: Risk of bias and quality assessment. a. Risk of bias graph: review authors' judgments about each risk of bias item presented as percentages across all included studies; b. Risk of bias summary: review authors' judgments about each risk of bias item for each included study. 
(a)

\begin{tabular}{|c|c|c|c|c|c|}
\hline \multirow[t]{2}{*}{ Study name } & \multirow[t]{2}{*}{ All-grade adverse events } & \multirow[b]{2}{*}{$\begin{array}{c}\text { Odds } \\
\text { ratio }\end{array}$} & \multicolumn{2}{|c|}{ Statistics for each study } & \multirow[b]{2}{*}{ p-Value } \\
\hline & & & $\begin{array}{c}\text { Lower } \\
\text { limit }\end{array}$ & $\begin{array}{c}\text { Upper } \\
\text { limit }\end{array}$ & \\
\hline Schlumberger 2015 & Dry mouth & 60.618 & 8.331 & 441.091 & 0.000 \\
\hline Schlumberger 2015 & Proteinuria & 33.370 & 2.022 & 550.607 & 0.014 \\
\hline Schlumberger 2015 & Rash & 29.594 & 1.789 & 489.579 & 0.018 \\
\hline Schlumberger 2015 & Dysgeusia & 29.025 & 7.009 & 120.201 & 0.000 \\
\hline Schlumberger 2015 & Fatigue or asthenia & 28.551 & 3.892 & 209.425 & 0.001 \\
\hline Schlumberger 2015 & Abdominal pain & 20.896 & 10.931 & 39.945 & 0.000 \\
\hline Schlumberger 2015 & Vomiting & 19.982 & 1.195 & 334.227 & 0.037 \\
\hline Schlumberger 2015 & Palmar-plantar erythrodysesthesia syndrome & 16.883 & 2.276 & 125.240 & 0.006 \\
\hline Schlumberger 2015 & Abdominal pain upper & 15.952 & 8.203 & 31.019 & 0.000 \\
\hline Schlumberger 2015 & Stomatitis & 14.383 & 1.930 & 107.211 & 0.009 \\
\hline Schlumberger 2015 & Diarrhea & 13.950 & 5.510 & 35.319 & 0.000 \\
\hline Schlumberger 2015 & Headache & 13.078 & 3.118 & 54.853 & 0.000 \\
\hline Schlumberger 2015 & Hypertension & 12.370 & 2.945 & 51.954 & 0.001 \\
\hline Schlumberger 2015 & Edema peripheral & 10.102 & 3.589 & 28.436 & 0.000 \\
\hline Schlumberger 2015 & Arthralgia & 8.571 & 4.512 & 16.280 & 0.000 \\
\hline Schlumberger 2015 & Anorexia & 7.793 & 4.320 & 14.058 & 0.000 \\
\hline Schlumberger 2015 & Myalgia & 7.271 & 2.200 & 24.027 & 0.001 \\
\hline Schlumberger 2015 & Dyspepsia & 6.084 & 2.834 & 13.063 & 0.000 \\
\hline Schlumberger 2015 & Dysphonia & 5.857 & 2.726 & 12.586 & 0.000 \\
\hline Schlumberger 2015 & Constipation & 4.362 & 2.503 & 7.600 & 0.000 \\
\hline Schlumberger 2015 & Nausea & 4.032 & 1.543 & 10.536 & 0.004 \\
\hline Schlumberger 2015 & Alopecia & 3.798 & 2.407 & 5.993 & 0.000 \\
\hline Schlumberger 2015 & Oropharyngeal pain & 3.774 & 1.440 & 9.894 & 0.007 \\
\hline Schlumberger 2015 & Pulmonary embolism & 3.150 & 1.190 & 8.339 & 0.021 \\
\hline Schlumberger 2015 & Hypocalcemia & 1.859 & 0.917 & 3.769 & 0.086 \\
\hline \multirow{2}{*}{ Schlumberger 2015} & Weight loss & 1.778 & 0.364 & 8.679 & 0.477 \\
\hline & & 7.876 & 5.709 & 10.865 & 0.000 \\
\hline
\end{tabular}

Odds ratio and $95 \% \mathrm{Cl}$

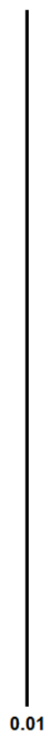

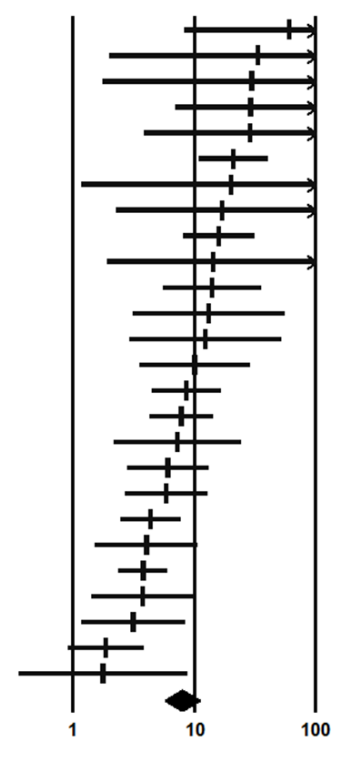

Favours A

Favours B

(b)

\begin{tabular}{|c|c|c|c|c|c|}
\hline \multirow[t]{2}{*}{$\underline{\text { Study name }}$} & \multirow[t]{2}{*}{ Grade more than 3 adverse events } & \multirow[b]{2}{*}{$\begin{array}{l}\text { Odds } \\
\text { ratio }\end{array}$} & \multicolumn{2}{|c|}{ Statistics for each study } & \multirow[b]{2}{*}{ p-Value } \\
\hline & & & $\begin{array}{l}\text { Lower } \\
\text { limit }\end{array}$ & $\begin{array}{l}\text { Upper } \\
\text { limit }\end{array}$ & \\
\hline Schlumberger 2015 & Hypertension & 30.596 & 9.488 & 98.671 & 0.000 \\
\hline Schlumberger 2015 & Proteinuria & 29.594 & 1.789 & 489.579 & 0.018 \\
\hline Schlumberger 2015 & Weight loss & 28.357 & 1.712 & 469.582 & 0.020 \\
\hline Schlumberger 2015 & Diarrhea & 23.511 & 1.413 & 391.265 & 0.028 \\
\hline Schlumberger 2015 & Anorexia & 15.408 & 0.912 & 260.345 & 0.058 \\
\hline Schlumberger 2015 & Stomatitis & 12.074 & 0.706 & 206.504 & 0.086 \\
\hline Schlumberger 2015 & Palmar-plantar erythrodysesthesia syndrome & 9.895 & 0.571 & 171.340 & 0.115 \\
\hline Schlumberger 2015 & Headache & 7.750 & 0.439 & 136.756 & 0.162 \\
\hline Schlumberger 2015 & Hypocalcemia & 7.750 & 0.439 & 136.756 & 0.162 \\
\hline Schlumberger 2015 & Vomiting & 5.639 & 0.309 & 102.766 & 0.243 \\
\hline Schlumberger 2015 & Myalgia & 4.596 & 0.246 & 86.013 & 0.307 \\
\hline Schlumberger 2015 & Fatigue or asthenia & 4.321 & 1.276 & 14.625 & 0.019 \\
\hline Schlumberger 2015 & Dysphonia & 3.561 & 0.183 & 69.452 & 0.402 \\
\hline Schlumberger 2015 & Nausea & 3.059 & 0.364 & 25.676 & 0.303 \\
\hline Schlumberger 2015 & Pulmonary embolism & 1.778 & 0.364 & 8.679 & 0.477 \\
\hline Schlumberger 2015 & Constipation & 1.514 & 0.061 & 37.430 & 0.800 \\
\hline Schlumberger 2015 & Dry mouth & 1.514 & 0.061 & 37.430 & 0.800 \\
\hline Schlumberger 2015 & Edema peripheral & 1.514 & 0.061 & 37.430 & 0.800 \\
\hline Schlumberger 2015 & Oropharyngeal pain & 1.514 & 0.061 & 37.430 & 0.800 \\
\hline Schlumberger 2015 & Rash & 1.514 & 0.061 & 37.430 & 0.800 \\
\hline \multirow[t]{2}{*}{ Schlumberger 2015} & Abdominal pain & 0.500 & 0.031 & 8.058 & 0.625 \\
\hline & & 6.226 & 3.772 & 10.279 & 0.000 \\
\hline
\end{tabular}

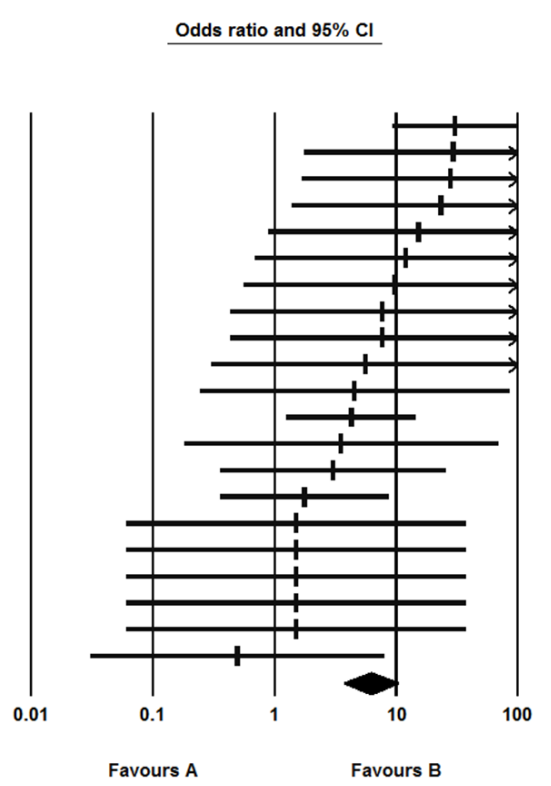

Favours A

Favours B

\section{Meta Analysis}

Figure 4: The odds ratios (ORs) of adverse events (AEs) in a controlled trial comparing lenvatinib and placebo. a. OR and $95 \%$ CIs of all-grade AEs using a random-effects model; b. OR and 95\% CIs of grade $\geq 3$ AEs using a fixed-effects model. 
tolerable with encouraging anti-tumor activity in patients with solid tumors.

The heterogeneity in our analysis could arise from different tumor types, the very heterogeneous study population with pre-treated disease and the ethnicity difference. In addition, there are several limitations of our study. Firstly, because lenvatinib is a relatively new drug, reports about it are few and are mostly phase I and II studies. Secondly, only one study provided the overall survival data, so prolonged follow-ups are needed. Thirdly, we did not perform subgroup analysis of melanoma and non-small-cell lung cancer because of lack of enough information.

In conclusion, lenvatinib has clinically meaningful benefits in survival outcomes of patients with thyroid cancer. The pooled analyses suggest that patients should be monitored for potential thrombocytopenia and increases in blood pressure, and dose reductions or delays or antihypertensive drugs are needed accordingly. Correct estimates of treatment-related toxicities and the efficacy of lenvatinib are fundamental to provide appropriate guidance and to conduct ongoing trials.

\section{MATERIALS AND METHODS}

\section{Search strategy}

We performed a literature search of PubMed, Medline, Embase, Web of Science and The Cochrane Library for all the relevant clinical trials on the safety and efficacy of lenvatinib (until April 26, 2016, 201). In order to ensure the completeness of the results, we expanded the search scope by using the search terms "lenvatinib" or "E7080" or "lenvima". We also carried out further searches for relevant unpublished trials in the clinical trial registry (http://www.clinicaltrials.gov). Papers in all languages were sought and translated where appropriate to reduce the chances of bias.

\section{Inclusion and exclusion criteria}

To be included in the analysis, patients must be diagnosed with histologically confirmed tumors, survival outcomes and toxicities were mandatory to be reported. All phase clinical trials were eligible for inclusion if they evaluated the side effects and efficacy of lenvatinib. Studies were excluded if they did not provide enough data for toxicities and survival outcomes. They were also excluded for which full-text reports were not available.

\section{Selection process and data extraction}

Two reviewers selected studies independently. Any disagreements were resolved through discussion with another author. We excluded those studies that clearly did not meet the inclusion criteria, and made efforts to rule out duplicated studies by comparing author lists, publication year, and the main contents if necessary. Articles with the same author(s) or medical center(s) were carefully reviewed and discussed for eligibility.

Data extracted from all eligible articles included the first author, year of publication, sample size, study phase, tumor type, treatment regime, progression-free survival (PFS), hazard ratio (HR) and adverse events. ADRs were graded using the National Cancer Institute (Washington, DC, USA) Common Toxicity Criteria, version 3.0.

\section{Data analysis}

We used patients' all-grade and grade $\geq 3$ Common Toxicity Criteria Adverse Events (CTCAE) counts to calculate the incidence rates of AEs and the corresponding 95\% confidence intervals (CIs). I-squared was calculated to test heterogeneity of the studies, and $\mathrm{I}^{2}>50 \%$ and $\mathrm{P}$ $\leq 0.1$ indicated strong heterogeneity between the studies. All the analysis was carried out using the software Comprehensive Meta-Analysis (CMA) program 2 (Biostat, Englewood, NJ) and Review manager 5.3 (Copenhagen, Sweden).

\section{Risk of bias and quality assessment}

To evaluate the risk of bias and quality of the studies, QUADAS-2 was used as a systematic review assessment method, which consisted of four key domains: patient selection, index test, reference standard and flow and timing [36]. Risk of bias was rated as high/low/ unclear. The assessment was measured using Review Manager 5.3 (Copenhagen, Sweden).

\section{CONFLICTS OF INTEREST}

All authors declare no conflicts of interest

\section{REFERENCES}

1. Verheul HM, Voest EE, Schlingemann RO. Are tumours angiogenesis-dependent. J Pathol. 2004; 202: 5-13.

2. Bergers G, Benjamin LE. Tumorigenesis and the angiogenic switch. Nat Rev Cancer. 2003; 3: 401-10.

3. Mazitschek R, Giannis A. Inhibitors of angiogenesis and cancer-related receptor tyrosine kinases. Curr Opin Chem Biol. 2004; 8: 432-41.

4. Alonso-Gordoa T, Díez JJ, Durán M, Grande E. Advances in thyroid cancer treatment: latest evidence and clinical potential. Ther Adv Med Oncol. 2015; 7: 22-38.

5. Okamoto K, Kodama K, Takase K, Sugi NH, Yamamoto Y, Iwata M, Tsuruoka A. Antitumor activities of the targeted multi-tyrosine kinase inhibitor lenvatinib (E7080) against RET gene fusion-driven tumor models. Cancer Lett. 2013; 340: 97-103. 
6. Matsui J, Yamamoto Y, Funahashi Y, Tsuruoka A, Watanabe T, Wakabayashi T, Uenaka T, Asada M. E7080, a novel inhibitor that targets multiple kinases, has potent antitumor activities against stem cell factor producing human small cell lung cancer H146, based on angiogenesis inhibition. Int J Cancer. 2008; 122: 664-71.

7. Matsui J, Funahashi Y, Uenaka T, Watanabe T, Tsuruoka A, Asada M. Multi-kinase inhibitor E7080 suppresses lymph node and lung metastases of human mammary breast tumor MDA-MB-231 via inhibition of vascular endothelial growth factor-receptor (VEGF-R) 2 and VEGF-R3 kinase. Clin Cancer Res. 2008; 14: 5459-65.

8. Glen H, Mason S, Patel H, Macleod K, Brunton VG. E7080, a multi-targeted tyrosine kinase inhibitor suppresses tumor cell migration and invasion. BMC Cancer. 2011; 11: 309.

9. Nair A, Lemery SJ, Yang J, Marathe A, Zhao L, Zhao H, Jiang X, He K, Ladouceur G, Mitra AK, Zhou L, Fox E, Aungst S, et al. FDA Approval Summary: Lenvatinib for Progressive, Radio-iodine-Refractory Differentiated Thyroid Cancer. Clin Cancer Res. 2015; 21:5205-8. doi: 10.1158/1078-0432.CCR-15-1377.

10. Schlumberger M, Jarzab B, Cabanillas ME, Robinson B, Pacini F, Ball DW, McCaffrey J, Newbold K, Allison R, Martins RG, Licitra LF, Shah MH, Bodenner D, et al. A Phase II Trial of the Multitargeted Tyrosine Kinase Inhibitor Lenvatinib (E7080) in Advanced Medullary Thyroid Cancer. Clin Cancer Res. 2016; 22:44-53. doi: 10.1158/1078-0432.CCR-15-1127.

11. Jasim S, Ozsari L, Habra MA. Multikinase inhibitors use in differentiated thyroid carcinoma. Biologics. 2014; 8: 281-91.

12. Carneiro RM, Carneiro BA, Agulnik M, Kopp PA, Giles FJ. Targeted therapies in advanced differentiated thyroid cancer. Cancer Treat Rev. 2015; 41: 690-8.

13. Boss DS, Glen H, Beijnen JH, Keesen M, Morrison R, Tait B, Copalu W, Mazur A, Wanders J, O'Brien JP, Schellens JH, Evans TR. A phase I study of E7080, a multitargeted tyrosine kinase inhibitor, in patients with advanced solid tumours. Br J Cancer. 2012; 106: 1598-604.

14. Hong DS, Kurzrock R, Wheler JJ, Naing A, Falchook GS, Fu S, Kim KB, Davies MA, Nguyen LM, George GC, Xu L, Shumaker R, Ren M, et al. Phase I Dose-Escalation Study of the Multikinase Inhibitor Lenvatinib in Patients with Advanced Solid Tumors and in an Expanded Cohort of Patients with Melanoma. Clin Cancer Res. 2015; 21: 4801-10.

15. Cabanillas ME, Schlumberger M, Jarzab B, Martins RG, Pacini F, Robinson B, McCaffrey JC, Shah MH, Bodenner DL, Topliss D, Andresen C, O'Brien JP, Ren M, et al. A phase 2 trial of lenvatinib (E7080) in advanced, progressive, radioiodine-refractory, differentiated thyroid cancer: A clinical outcomes and biomarker assessment. Cancer. 2015; 121: 2749-56.

16. Molina AM, Hutson TE, Larkin J, Gold AM, Wood $\mathrm{K}$, Carter D, Motzer R, Michaelson MD. A phase $1 \mathrm{~b}$ clinical trial of the multi-targeted tyrosine kinase inhibitor lenvatinib (E7080) in combination with everolimus for treatment of metastatic renal cell carcinoma (RCC). Cancer Chemother Pharmacol. 2014; 73: 181-9.

17. Yamada K, Yamamoto N, Yamada Y, Nokihara H, Fujiwara Y, Hirata T, Koizumi F, Nishio K, Koyama N, Tamura T. Phase I dose-escalation study and biomarker analysis of E7080 in patients with advanced solid tumors. Clin Cancer Res. 2011; 17: 2528-37.

18. Hong DS, Kurzrock R, Falchook GS, Andresen C, Kwak J, Ren M, Xu L, George GC, Kim KB, Nguyen LM, O'Brien JP, Nemunaitis J. Phase 1b study of lenvatinib (E7080) in combination with temozolomide for treatment of advanced melanoma. Oncotarget. 2015; 6:43127-34. doi: 10.18632/ oncotarget.5756.

19. Motzer RJ, Hutson TE, Glen H, Michaelson MD, Molina A, Eisen T, Jassem J, Zolnierek J, Maroto JP, Mellado B, Melichar B, Tomasek J, Kremer A, et al. Lenvatinib, everolimus, and the combination in patients with metastatic renal cell carcinoma: a randomised, phase 2, open-label, multicentre trial. Lancet Oncol. 2015; 16: 1473-82.

20. Nakamichi S, Nokihara H, Yamamoto N, Yamada Y, Honda K, Tamura Y, Wakui H, Sasaki T, Yusa W, Fujino $\mathrm{K}$, Tamura T. A phase 1 study of lenvatinib, multiple receptor tyrosine kinase inhibitor, in Japanese patients with advanced solid tumors. Cancer Chemother Pharmacol. 2015; 76: 1153-61.

21. Ikeda M, Okusaka T, Mitsunaga S, Uneo H, Tamai T, Suzuki T, Hayato S, Kadowaki T, Okita K, Kumada H. Safety and pharmacokinetics of lenvatinib in patients with advanced hepatocellular carcinoma. Clin Cancer Res. 2016; 22:1385-94. doi: 10.1158/1078-0432.CCR-15-1354.

22. Schlumberger M, Tahara M, Wirth LJ, Robinson B, Brose MS, Elisei R, Habra MA, Newbold K, Shah MH, Hoff AO, Gianoukakis AG, Kiyota N, Taylor MH, et al. Lenvatinib versus placebo in radioiodine-refractory thyroid cancer. N Engl J Med. 2015; 372: 621-30.

23. Dubbelman AC, Rosing $\mathrm{H}$, Nijenhuis C, Huitema AD, Mergui-Roelvink M, Gupta A, Verbel D, Thompson G, Shumaker R, Schellens JH, Beijnen JH. Pharmacokinetics and excretion of (14)C-lenvatinib in patients with advanced solid tumors or lymphomas. Invest New Drugs. 2015; 33: 233-40.

24. Shumaker RC, Aluri J, Fan J, Martinez G, Thompson GA, Ren M. Effect of rifampicin on the pharmacokinetics of lenvatinib in healthy adults. Clin Drug Investig. 2014; 34: 651-9.

25. Nishio M, Horai T, Horiike A, Nokihara H, Yamamoto N, Takahashi T, Murakami H, Yamamoto N, Koizumi F, Nishio K, Yusa W, Koyama N, Tamura T. Phase 1 study of lenvatinib combined with carboplatin and paclitaxel in patients with non-small-cell lung cancer. Br J Cancer. 2013; 109: 538-44.

26. Schlumberger M, Jarzab B, Cabanillas ME, Robinson B, Pacini F, Ball DW, McCaffrey J, Newbold K, Allison 
R, Martins RG, Licitra LF, Shah MH, Bodenner D, et al. A Phase II Trial of the Multitargeted Tyrosine Kinase Inhibitor Lenvatinib (E7080) in Advanced Medullary Thyroid Cancer. Clin Cancer Res. 2016; 22: 44-53.

27. Lin Z, Yang Y, Huang Y, Liang J, Lu F, Lao X. Vascular endothelial growth factor receptor tyrosine kinase inhibitors versus bevacizumab in metastatic colorectal cancer: A systematic review and meta-analysis. Mol Clin Oncol. 2015; 3: 959-67.

28. Zhang X, Qin Y, Li H, Bai C, Zhu T, Xu J, Wu C, Wu M, Wang C, Song H, Wei L, He J. Efficacy and safety of vandetanib, a dual VEGFR and EGFR inhibitor, in advanced non-small-cell lung cancer: a systematic review and meta-analysis. Asian Pac J Cancer Prev. 2011; 12: 2857-63.

29. Keizer RJ, Gupta A, Mac GMR, Jansen M, Wanders J, Beijnen JH, Schellens JH, Karlsson MO, Huitema AD. A model of hypertension and proteinuria in cancer patients treated with the anti-angiogenic drug E7080. J Pharmacokinet Pharmacodyn. 2010; 37: 347-63.

30. Robinson ES, Matulonis UA, Ivy P, Berlin ST, Tyburski K, Penson RT, Humphreys BD. Rapid development of hypertension and proteinuria with cediranib, an oral vascular endothelial growth factor receptor inhibitor. Clin J Am Soc Nephrol. 2010; 5: 477-83.

31. Zhu X, Wu S, Dahut WL, Parikh CR. Risks of proteinuria and hypertension with bevacizumab, an antibody against vascular endothelial growth factor: systematic review and meta-analysis. Am J Kidney Dis. 2007; 49: 186-93.
32. Chen J, Lu Y, Zheng Y. Incidence and risk of hypertension with bevacizumab in non-small-cell lung cancer patients: a meta-analysis of randomized controlled trials. Drug Des Devel Ther. 2015; 9: 4751-60.

33. Minatsuki S, Miura I, Yao A, Abe H, Muraoka H, Tanaka M, Imamura T, Inaba T, Maki H, Hatano M, Kinugawa K, Yao T, Fukayama M, et al. Platelet-derived growth factor receptor-tyrosine kinase inhibitor, imatinib, is effective for treating pulmonary hypertension induced by pulmonary tumor thrombotic microangiopathy. Int Heart J. 2015; 56: 245-8.

34. Bergsten E, Uutela M, Li X, Pietras K, Ostman A, Heldin $\mathrm{CH}$, Alitalo K, Eriksson U. PDGF-D is a specific, proteaseactivated ligand for the PDGF beta-receptor. Nat Cell Biol. 2001; 3: 512-6.

35. Horinouchi H, Yamamoto N, Nokihara H, Horai T, Nishio M, Ohyanagi F, Horiike A, Nakagawa K, Terashima M, Okabe T, Kaneda H, McKee MD, Carlson DM, et al. A phase 1 study of linifanib in combination with carboplatin/ paclitaxel as first-line treatment of Japanese patients with advanced or metastatic non-small cell lung cancer (NSCLC). Cancer Chemother Pharmacol. 2014; 74: 37-43.

36. Whiting PF, Rutjes AW, Westwood ME, Mallett S, Deeks JJ, Reitsma JB, Leeflang MM, Sterne JA, Bossuyt PM. QUADAS-2: a revised tool for the quality assessment of diagnostic accuracy studies. Ann Intern Med. 2011; 155: 529-36. 